\title{
Should a single blastocyst transfer policy be a clinical decision or should it depend on the embryological evaluation on day 3?
}

\author{
Dominic Stoop*, Lisbet Van Landuyt, Etienne Van den Abbeel, Michel Camus, Greta Verheyen and Paul Devroey
}

\begin{abstract}
Background: Single blastocyst transfer has the advantage of maximizing the fresh single pregnancy rate. However, in patients with a low number of good quality embryos on day 3, it remains unclear whether immediate embryo transfer or further embryo culture with blastocyst transfer is the most preferable option.

Methods: A retrospective cohort study was carried out in which the outcome of 590 fresh in vitro fertilization (IVF) cycles over a 15 months period and their cryo cycles were analyzed. A total of 341 patients cycles had an elective day 5 strategy independent of intermediate embryo evaluation while another 249 patients underwent a day 5 embryo transfer only if at least four embryos were available on day 3. Blastocyst vitrification was performed using a closed high security system.

Results: Demographics, stimulation parameters and embryological data were comparable in the two groups. Patients in the elective day 5 group had a lower fresh transfer rate $(90.62 \%$ vs. $95.18 \%, p<0.05)$ as compared to patients with a day 3 or day 5 embryo transfer policy. No difference was observed in the fresh live birth rate and multiple pregnancy rate per initiated cycle (32.84\% vs. $28.92 \% ; 1.17 \%$ vs $0 \%)$ The projected cumulative ongoing pregnancy rate compensating for double counting in case subjects have more than one pregnancy is not different (42.58\% vs. $39.84 \%)$.

Conclusions: Despite lower fresh transfer rates, elective single blastocyst transfer yields a similar projected cumulative ongoing pregnancy rate as in a policy with cleavage stage or blastocyst transfer depending on a good quality embryo count on day 3.
\end{abstract}

\section{Background}

Single embryo transfer (SET) has been advocated as a strategy to reduce the frequency of multiple births after in vitro fertilization [1]. It has been established that the significantly lower pregnancy rate achieved after single cleavage stage embryo transfer as compared to a double embryo transfer can be compensated by pregnancies resulting from the first thawed cycle [2]. This finding underscores the important role of cryopreservation in enhancing the total reproductive potential of a single cycle [3]. The introduction of the vitrification technique as an efficient cryopreservation technique promises to

\footnotetext{
* Correspondence: dominic.stoop@uzbrussel.be

Centre for Reproductive Medicine, University Hospital, Vrije Universiteit Brussel (Free University of Brussels), Laarbeeklaan 101, 1090 Brussels, Belgium
}

further increase the impact of cryo-technology on the cycle specific pregnancy rates.

Fresh pregnancy rates can be optimized by performing blastocyst transfer as such approach yields higher live birth rates than single cleavage stage embryo transfer $[4,5]$. However, a systematic blastocyst transfer policy holds the risk of cancelled embryo transfers, as extended embryo culture may be unsuccessful. Morphological scoring of embryo quality on day 3 may therefore offer a tool to identify patients that would potentially benefit from further embryo culture [6]. The threshold of four good embryos on the third day appeared to be a reassuring indication that the patient will undergo an embryo transfer [6].

The aim of present study is to compare two approaches, both aimed at a single blastocyst transfer. The outcome after elective blastocyst transfer was compared with the outcome of a blastocyst transfer or a cleavage stage transfer 
depending on whether the criteria of four good embryos on the third day were met. Moreover, the additional cryopregnancies and projected cryo-pregnancies through blastocyst vitrification have been evaluated.

There is an ongoing debate on the microbiological safety of cryopreservation since the report of a hepatitis $B$ transmission from contaminated cryopreserved bone marrow and reports on potential contamination of reproductive cells in experimental conditions $[7,8]$. Although no clinical reports of contamination have been reported for reproductive cells and tissues, research is ongoing aimed at eliminating such risks. In this study we evaluate the effectiveness of blastocyst vitrification performed with a high security vitrification system preventing all contamination risk during vitrification and storage.

\section{Methods \\ Study design}

A retrospective cohort study was performed. All fresh IVF or ICSI treatment cycles were performed in a 15 months period from March 2008 until June 2009. All cycles where the physicians' initial treatment plan envisaged eSET with either a fixed day 5 transfer or with a day 3 or day 5 transfer depending on the embryo quality on day 3 were included. Only the first treatment cycle was considered in case patients were undergoing multiple attempts.

\section{Embryo culture, evaluation and selection day of transfer} Sperm preparation, IVF/ICSI procedures and embryo culture were carried out as described by Van Landuyt et al. [9]. Oocytes and embryos were cultured in sequential media and cultured at $37^{\circ} \mathrm{C}$ in an atmosphere of $6 \%$ $\mathrm{CO}_{2}, 5 \% \mathrm{O}_{2}$ and $89 \% \mathrm{~N}_{2}$. Fertilization was assessed $16-$ $19 \mathrm{~h}$ after insemination or injection. On the morning of day 3 embryos were transferred from cleavage medium to blastocyst medium. Embryo quality was assessed daily was scored as described by Papanikolaou et al., [6] according to the following parameters: number of blastomeres, rate of fragmentation, multinucleation of the blastomeres and early compaction. Blastocyst quality on day 5/6 was assessed according to the criteria of Gardner and Schoolcraft [10].

\section{Embryo cryopreservation procedures}

For patients receiving transfer on day 3, supernumerary cleavage stage embryos were cryopreserved and thawed using standard slow freezing as described by Van den Abbeel et al., (2005). Blastocyst vitrification was performed using closed CBS-VIT High Security (HS) straws (Cryo Bio system) in combination with DMSO-EG-S as the cryoprotectants (Irvine Scientific ${ }^{\mathrm{R}}$ Freeze Kit). The vitrification procedure was carried out at room temperature (between
22 and $27^{\circ} \mathrm{C}$ ). Blastocysts were vitrified one by one. The blastocyst was first incubated for $2 \mathrm{~min}$ in a $50 \mu \mathrm{l}$ droplet of HEPES-buffered culture medium. Then, the blastocyst was brought in a $50 \mu \mathrm{l}$ droplet of equilibration solution (ES) containing 7.5\% (v/v) DMSO and 7.5\% (v/v) ethylene glycol and incubated for $10 \mathrm{~min}$. The blastocyst was then transferred consecutively into four $25-\mu \mathrm{l}$ droplets with vitrification solution (VS) containing 15\% (v/v) DMSO and $15 \%(\mathrm{v} / \mathrm{v})$ ethylene glycol. The blastocyst was incubated for $5 \mathrm{sec}$ in droplet 1 and 2 and for $10 \mathrm{sec}$ in the $3^{\text {rd }}$ droplet. The blastocyst was then transferred to the fourth droplet and immediately loaded onto the CBS-HS straw. The straw was heat sealed and plunged into liquid nitrogen. The total time needed to vitrify the blastocyst starting from VS droplet 1 to the loading of the straw and plunging into liquid nitrogen did not exceed $90 \mathrm{sec}$.

On the day of transfer blastocysts were warmed one by one until one blastocyst was available for transfer. Blastocysts were warmed randomly, independent of the blastocyst stage or ICM/TE quality prior to vitrification. For warming, the Irvine Scientific ${ }^{\mathrm{R}}$ Thaw Kit was used. A Petri-dish containing two $25 \mu$ l droplets with thawing solution (TS, $1 \mathrm{M}$ sucrose in Hepes buffered HTF medium supplemented with $20 \%$ DSS) was kept at $37^{\circ} \mathrm{C}$. For warming the straw, the straw was transferred from the $\mathrm{LN}_{2}$ storage container to a transport dewar filled with liquid nitrogen. After cutting the straw and pulling the capillary from the straw, the gutter was placed in the first droplet with TS and the blastocyst was released from the gutter and kept at room temperature. The blastocyst was incubated for two times $1 \mathrm{~min}$ at room temperature in the two TS droplets. The blastocyst was then transferred to the first of two dilution solution droplets of $25 \mu \mathrm{l}$ (DS; $0.5 \mathrm{M}$ sucrose in Hepes buffered HTF medium supplemented with 20\% DSS) and after that incubated for $2 \mathrm{~min}$ in a second DS droplet. Finally, the blastocyst was washed in 3 droplets $(25 \mu \mathrm{l})$ of washing solution (Hepes buffered HTF medium supplemented with 20\% DSS), each for $3 \mathrm{~min}$. The blastocyst was transferred to a culture dish with blastocyst medium to assess its morphological survival. If the blastocyst was severely or completely damaged, a new one was warmed immediately. If the blastocyst was fully intact or showed moderate damage, expansion and re-expansion was assessed 1-2 hours later. If the morphological quality of the blastocyst was regressing or no signs of re-expansion were present, a second blastocyst was warmed until one blastocyst was suitable for transfer i.e. with at least moderate survival and expansion/re-expansion of the blastocyst

\section{Statistical analysis}

All data management and statistical analysis were performed using the Statistical Package for the Social Sciences version 17.0 (SPSS Inc. Chicago, OL, USA). 
Student't t-test were performed on continuous variables to determine differences in mean scores and standard deviation (SD). Categorical variables were analyzed using Chi square analysis. A significant level of 0.05 was accepted throughout.

\section{Results}

\section{Patient demographics and stimulation characteristics} (Table 1)

Overall 590 treatment cycles have been performed during the observation period with 341 (58\%) in the fixed day 5 group and $247(42 \%)$ in the day 3 or day 5 group. The two groups were comparable with regard to age, rank of trial, duration of stimulation and the total dose of gonadotrophins administered.

\section{Fresh embryo transfer: embryology data (Table 2)}

Overall, no differences were observed in the total number of cumulus-oocyte complexes, MII oocytes or 2PN oocytes. However, a significantly lower number of cumulus-oocyte complexes, MII oocytes and 2PN oocytes were available in the subgroup of patients with a transfer on day 3. Patients with the fixed day 5 approach had a lower subjects' fresh transfer rate $(90.62 \%$ vs. $95.18 \%, \mathrm{p}<0.05)$ but the embryo fresh transfer rate and the embryo cryopreservation rate was similar. For patients in the day 3 or day 5 group, a higher embryo fresh transfer rate and a lower embryo cryopreservation rate were observed in patients with a transfer on day 3 . The data reported separately for the day 3 and day 5 subgroups only included cycles with embryo transfer as cancelled cycles $(n=12)$ cannot be allocated to either one of the two subgroups.

\section{Fresh embryo transfer: outcome (Table 3)}

No differences were observed in terms of clinical pregnancy rate, ongoing pregnancy rate or live birth rate between the fixed day 5 and the day 3 or day 5 transfer policies. These outcome parameters also did not differ between the two subgroups within the day 3 or day 5 transfer policy. Multiple pregnancies were only observed in the fixed day 5 approach (1.29\%) but did not statistically higher than in the other transfer approach group.

\section{Frozen embryo transfer: embryology data (Table 4)}

An overall embryo transfer rate of $69.9 \%$ was observed in the fixed blastocyst group which was not significantly different from the overall embryo transfer rate of $65.5 \%$ was observed for day 3 and day 5 embryos combined in the other group. The embryo transfer rate was statistically higher in the day 5 subgroup as compared to the day 3 subgroup $(76.5 \%$ vs. $46.4 \%, \mathrm{p}<0.001)$. No differences were observed in the transfer specific or subject specific clinical pregnancy rates.

\section{Frozen embryo transfer: outcome (Table 5)}

Single embryo transfer was performed in $83 \%$ of all frozen blastocyst transfers in the fixed day 5 group. Single blastocyst transfers in the day 5 subgroup of the day 3 or day 5 group counted for $85.6 \%$ of the cycles which was significantly more than the $50 \%$ SET observed in the day 3 subgroup. The ongoing pregnancy rate per frozen embryo transfer in the fixed day 5 groups was $14.04 \%$, which was not different from the overall ongoing pregnancy rate of the day 3 or day 5 group (14.63\%). Multiple pregnancy rates were not different between the different approaches.

\section{Summary and projected outcome (Table 6)}

The clinical and ongoing pregnancy rate per thawed embryo was not different between the two groups. Neither was the projected cumulative clinical and ongoing pregnancy rate different between the elective day 5 group $(44.12 \% ; 42.58 \%)$ versus the day 3 or day 5 group (44.04\%; 39.84\%).

\section{Discussion}

This study has shown that a single blastocyst transfer policy can be decided before the initiation of the in

Table 1 Patient demographics and stimulation characteristics

\begin{tabular}{|c|c|c|c|c|}
\hline & eSET with elective day 5 transfer & \multicolumn{2}{|c|}{ eSET with transfer day 3 or 5} & Statistical significance \\
\hline No. subjects & 341 & \multicolumn{2}{|c|}{249} & - \\
\hline Age (years) & $29.94(3.54)$ & \multicolumn{2}{|c|}{$31.20(3.18)$} & NS \\
\hline Rank of trial & $0.29(0.61)$ & \multicolumn{2}{|c|}{$0.39(0.68)$} & NS \\
\hline Days of stimulation & $9.53(2.09)$ & \multicolumn{2}{|c|}{$9.83(2.22)$} & NS \\
\hline \multirow[t]{2}{*}{ Total gonadotropin dose (IU) } & $1745.95(632.93)$ & \multicolumn{2}{|c|}{$1857.56(685.55)$} & NS \\
\hline & & Day 3 & Day 5 & \\
\hline No. subjects & & 135 & 102 & \\
\hline Age (years) & & $31.24(3.23)$ & $31.30(3.11)$ & NS \\
\hline Rank of trial & & $0.36(0.66)$ & $0.44(0.73)$ & NS \\
\hline Days of stimulation & & $9.94(2.35)$ & $9.78(1.98)$ & \\
\hline Total gonadotropin dose ((IU) & & $1907.56(671.37)$ & $1800.17(704.74)$ & NS \\
\hline
\end{tabular}


Table 2 Embryology data: fresh embryo transfer

\begin{tabular}{|c|c|c|c|c|}
\hline & $\begin{array}{l}\text { eSET with elective day } 5 \\
\text { transfer }\end{array}$ & \multicolumn{2}{|c|}{ eSET with transfer day 3 or 5} & $\begin{array}{l}\text { Statistical } \\
\text { significance }\end{array}$ \\
\hline \multicolumn{5}{|c|}{$\begin{array}{l}\text { Proportion IVF/ICSI as in vitro fertilization procedure } \\
(\%)\end{array}$} \\
\hline IVF & $44 / 341(12.90)$ & \multicolumn{2}{|c|}{ 24/247 (9.72) } & NS \\
\hline ICSI & $297 / 341(87.10)$ & \multicolumn{2}{|c|}{$223 / 247(90.28)$} & NS \\
\hline Mean No. cumulus-oocyte complexes (SD) & $12.33(7.23)$ & \multicolumn{2}{|c|}{$11.66(7.35)$} & NS \\
\hline Mean No. metaphase II oocytes & $8.50(6.26)$ & \multicolumn{2}{|c|}{$8.27(6.46)$} & NS \\
\hline Mean No. two pronuclei oocytes & $7.46(5.03)$ & \multicolumn{2}{|c|}{7.11 (4.95) } & NS \\
\hline Embryos on day 3 & 2393 & \multicolumn{2}{|c|}{$1690^{1}$} & - \\
\hline Embryos on day 5 & 1619 & \multicolumn{2}{|c|}{$816^{2}$} & \\
\hline Embryos transferred fresh & 309 & \multicolumn{2}{|c|}{237} & - \\
\hline Embryo fresh transfer rate (\%) & 309/1619 (19.09) & \multicolumn{2}{|c|}{$237 / 1390^{3}(17.05)$} & NS \\
\hline Subjects with fresh transfer & 309 & \multicolumn{2}{|c|}{237} & - \\
\hline Subjects fresh transfer rate (\%) & $309 / 341(90.62)$ & \multicolumn{2}{|c|}{$237 / 249(95.18)$} & $<0.05$ \\
\hline Embryos cryopreserved & 640 & \multicolumn{2}{|c|}{$560^{4}$} & - \\
\hline Embryo cryopreservation rate (\%) & $640 / 1619(39.53)$ & \multicolumn{2}{|c|}{$560^{4} / 1465^{1}(38.22)$} & NS \\
\hline Subjects with cryopreservation & 191 & \multicolumn{2}{|c|}{$166^{5}$} & - \\
\hline \multirow[t]{2}{*}{ Subjects cryopreservation rate (\%) } & $191 / 309(61.81)$ & \multicolumn{2}{|c|}{$166 / 237(70.04)$} & 0.05 \\
\hline & & Day 3 & Day 5 & \\
\hline \multicolumn{5}{|c|}{$\begin{array}{l}\text { Proportion IVF/ICSI as in vitro fertilization procedure } \\
(\%)\end{array}$} \\
\hline IVF & & 12/135 (8.89) & 10/102 (9.80) & NS \\
\hline ICSI & & $\begin{array}{c}123 / 135 \\
(91.11)\end{array}$ & $92 / 102(90.20)$ & NS \\
\hline Mean No. cumulus-oocyte complexes (SD) & & $9.18(5.92)$ & $14.52(6.25)$ & $<0.05$ \\
\hline Mean No. metaphase II oocytes (SD) & & $6.00(4.31)$ & $11.01(6.08)$ & $<0.05$ \\
\hline Mean No. two pronuclei oocytes (SD) & & $4.73(3.00)$ & $10.05(4.06)$ & $<0.05$ \\
\hline Embryos on day 3 & & 615 & 1000 & - \\
\hline Embryos on day 5 & & NA & 775 & - \\
\hline Embryos transferred fresh & & 135 & 102 & - \\
\hline Embryo fresh transfer rate (\%) & & $\begin{array}{c}135 / 615 \\
(21.95)\end{array}$ & $\begin{array}{c}102 / 775 \\
(13.16)\end{array}$ & $<0.001$ \\
\hline Embryos cryopreserved & & 154 & 363 & \\
\hline Embryo cryopreservation rate (\%) & & $\begin{array}{c}154 / 615 \\
(25.04)\end{array}$ & $\begin{array}{c}363 / 775 \\
(46.84)\end{array}$ & $<0.001$ \\
\hline Subjects with cryopreservation & & 80 & 84 & - \\
\hline Subjects cryopreservation rate (\%) & & $80 / 135(59.26)$ & $84 / 102(82.35)$ & $<0.001$ \\
\hline
\end{tabular}

${ }^{1}$ including 75 day 3 embryos from 6 subjects without fresh transfer.

${ }^{2}$ including 41 day 5 embryos from 2 subjects without fresh transfer.

${ }^{3}$ available day 3 embryos for subjects with fresh day 3 transfer and available day 5 embryos for subjects with fresh day 5 transfer.

${ }^{4}$ including 43 embryo cryopreserved on day 3 and 5 in subjects without fresh transfer.

${ }^{5}$ including 2 subjects with cryopreserved embryos without fresh transfer.

vitro fertilization (IVF) cycle. A day 3 or day 5 embryo transfer policy based on day 3 embryo evaluation does not increase cycle outcome. It merely divides the patient population based on their ovarian response and reduces the risks of a cancelled embryo transfer.

Randomized controlled trials have shown that a blastocyst transfer policy results in a significantly higher live birth rate $[5,11]$ However, the effect of a fresh blastocyst transfer policy on the cumulative pregnancy rate, including cryo-cycles has not been evaluated in these trials.
A recent prospective trial by Guerif et al., [12] did include embryo cryopreservation by slow freezing technique when comparing a single day 2 with a single blastocyst transfer policy. Although blastocyst transfer yielded a higher live birth rate in the fresh cycles, it appeared to result in the same cumulative pregnancy rate after adding cryo-cycles. The authors concluded that improvements in blastocyst cryopreservation were needed in order to claim superiority of the blastocyst transfer policy. The implementation of the vitrification technique may provide such 
Table 3 Fresh embryo transfer outcome

\begin{tabular}{|c|c|c|c|c|c|}
\hline & \multicolumn{2}{|c|}{ eSET with elective day 5 transfer } & \multicolumn{2}{|c|}{ eSET with transfer day 3 or 5} & \multirow{2}{*}{$\frac{\text { Statistical significance }}{\text { /cycle }}$} \\
\hline & /cycle & /ET & /cycle & /ET & \\
\hline Pregnancy rate (positive hCG) & $141 / 341(41.35)$ & $141 / 309(45.63)$ & $95 / 249(38.15)$ & 95/237 (40.08) & 0.45 \\
\hline Clinical pregnancy rate & $121 / 341(35.48)$ & 121/309 (39.16) & 79/249 (31.73) & 79/237 (33.30) & 0.38 \\
\hline Ongoing pregnancy rate & $118 / 341(34.60)$ & 118/309 (38.19) & $73 / 249(29.32)$ & 73/237 (30.80) & 0.18 \\
\hline \multicolumn{6}{|l|}{ Pregnancy loss } \\
\hline Miscarriage & 14 & 14 & 10 & 10 & - \\
\hline Ectopic pregnancy & 5 & 5 & 1 & 1 & - \\
\hline Live birth rate & $112 / 341(32.84)$ & $112 / 309(36.25)$ & $72 / 249(28.92)$ & 72/237 (30.38) & 0.32 \\
\hline \multirow[t]{2}{*}{ Mulitple pregnancy rate } & 4/341 (1.17) & 4/309 (1.29) & 0/249 (0) & 0/237 (0) & 0.16 \\
\hline & & & Day 3/cycle (\%) & Day 5/cycle (\%) & \\
\hline Pregnancy rate (positive hCG) & & & 47/135 (34.81) & 48/102 (47.06) & 0.08 \\
\hline Clinical pregnancy rate & & & $39 / 135(28.89)$ & 40/102 (39.26) & 0.10 \\
\hline Ongoing pregnancy rate & & & $36 / 135$ 26.67) & $37 / 102(36.27)$ & 0.16 \\
\hline \multicolumn{6}{|l|}{ Pregnancy loss } \\
\hline Miscarriage & & & 6 & 4 & - \\
\hline Ectopic pregnancy & & & 1 & 0 & - \\
\hline Live birth rate & & & $36 / 135(26.67)$ & 36/102 (35.29) & 0.16 \\
\hline Mulitple pregnancy rate & & & 0/135 (0) & 0/102 (0) & 1 \\
\hline
\end{tabular}

effect, however it would also further increase cleavage stage cryopreservation outcome.

A meta analysis concludes that vitrification appears to be associated with a significantly higher postthawing survival rate than slow freezing [13]. The superiority of vitrification is also illustrated by comparing vitrification outcome in this study with previously published data on blastocyst slow-freezing at the same centre [14]. A reported embryo transfer rate ranging between $48.9 \%$ and $52.2 \%$ after slow freezing [14] has been substantially increased to rates varying between $69.9 \%$ and $76.5 \%$ after vitrification.

Table 4 Efficiency of cryopreservation and pregnancy potential of surviving embryos

\begin{tabular}{|c|c|c|c|c|}
\hline & eSET with elective day 5 transfer & \multicolumn{2}{|c|}{ eSET with transfer day 3 or 5} & Statistical significance \\
\hline No. of embryos cryopreserved & 640 & \multicolumn{2}{|c|}{517} & \\
\hline No. of thawing cycles & 188 & \multicolumn{2}{|c|}{143} & \\
\hline No. of embryos thawed & 286 & \multicolumn{2}{|c|}{229} & \\
\hline No. of embryos surviving & 200 & \multicolumn{2}{|c|}{150} & \\
\hline Transferrable survival rate & 200/286 (69.9) & \multicolumn{2}{|c|}{$150 / 229(65.5)$} & NS \\
\hline Subjects with thawed transfers & 109 & \multicolumn{2}{|c|}{71} & \\
\hline Total number of thawed transfers & 171 & \multicolumn{2}{|c|}{125} & \\
\hline Total number of clinical pregnancies & 26 & \multicolumn{2}{|c|}{21} & \\
\hline Transfer specific pregnancy rate ${ }^{1}$ & 26/171 (15.2) & \multicolumn{2}{|c|}{$21 / 125(16.8)$} & NS \\
\hline \multirow[t]{2}{*}{ Subject specific pregnancy rate ${ }^{2}$} & 26/109 (23.9) & \multicolumn{2}{|c|}{ 21/71 (29.6) } & NS \\
\hline & & Day 3 & Day 5 & \\
\hline No. of embryos cryopreserved & & 154 & 363 & \\
\hline No. of thawing cycles & & 41 & 102 & \\
\hline No. of embryos thawed & & 84 & 145 & \\
\hline No. of embryos surviving & & 39 & 111 & \\
\hline Transferrable survival rate & & $39 / 84(46.4)$ & $111 / 145(76.5)$ & $<0.001$ \\
\hline Subjects with thawed transfers & & 26 & 45 & \\
\hline Total number of thawed transfers & & 28 & 97 & \\
\hline Total number of clinical pregnancies & & 4 & 17 & \\
\hline Transfer specific pregnancy rate ${ }^{1}$ & & $4 / 28(14.3)$ & $17 / 97(17.5)$ & NS \\
\hline Subject specific pregnancy rate ${ }^{2}$ & & $4 / 26(15.4)$ & $17 / 45(37.8)$ & 0.05 \\
\hline
\end{tabular}

${ }^{1}$ Total number of pregnancies/total number of thawed transfers.

${ }^{2}$ Total number of pregnancies/number of subjects with thawed transfers. 
Table 5 Frozen embryo transfer outcome

\begin{tabular}{|c|c|c|c|c|c|c|c|}
\hline & \multicolumn{2}{|c|}{$\begin{array}{l}\text { eSET with elective day } 5 \\
\text { transfer }\end{array}$} & \multicolumn{4}{|c|}{ eSET with transfer day 3 or 5} & \multirow[t]{2}{*}{$\begin{array}{r}\text { Statistical } \\
\text { significance }\end{array}$} \\
\hline & $\begin{array}{c}\text { /cycle }(n= \\
188)\end{array}$ & $/ E T(n=171)$ & \multicolumn{2}{|c|}{ /cycle $(n=143)$} & \multicolumn{2}{|c|}{$/ \mathrm{ET}(\mathrm{n}=123)$} & \\
\hline Positive hCG (\%) & $34 / 188(18.06)$ & $\begin{array}{l}34 / 171 \\
(19.88)\end{array}$ & \multicolumn{2}{|c|}{$30 / 143(21.00)$} & \multicolumn{2}{|c|}{$30 / 123(24.39)$} & NS \\
\hline Clinical pregnancy rate (\%) & 26/188 (13.83) & $\begin{array}{l}26 / 171 \\
(15.20)\end{array}$ & \multicolumn{2}{|c|}{ 21/143 (14.69) } & \multicolumn{2}{|c|}{ 21/123 (17.07) } & NS \\
\hline $\begin{array}{l}\text { Ongoing pregnancy rate } \\
\text { (\%) }\end{array}$ & 24/188 (12.77) & $\begin{array}{l}24 / 171 \\
(14.04)\end{array}$ & \multicolumn{2}{|c|}{ 18/143 (12.56) } & \multicolumn{2}{|c|}{ 18/123 (14.63) } & NS \\
\hline \multicolumn{8}{|l|}{ Pregnancy loss } \\
\hline Miscarriage & \multicolumn{2}{|c|}{$2 / 26(7.69)$} & \multicolumn{4}{|c|}{$3 / 20(20)$} & NS \\
\hline Ectopic pregnancy & \multicolumn{2}{|c|}{0} & \multicolumn{4}{|c|}{$1 / 30(3.33)$} & NS \\
\hline Single embryo transfer (\%) & \multicolumn{2}{|c|}{ 142/171 (83.0) } & \multicolumn{4}{|c|}{$96 / 123(78.05)$} & NS \\
\hline \multirow[t]{3}{*}{ Multiple pregnancy rate (\%) } & \multicolumn{2}{|c|}{ 2/24 (8.33) } & \multicolumn{4}{|c|}{$1 / 18(5.56)$} & NS \\
\hline & & & \multicolumn{2}{|c|}{ Day 3} & \multicolumn{2}{|c|}{ Day 5} & \\
\hline & & & $\begin{array}{l}\text { /cycle }(n= \\
41)\end{array}$ & $/ E T(n=26)$ & $\begin{array}{c}\text { /cycle }(\mathrm{n}= \\
102)\end{array}$ & $/ \mathrm{ET}(\mathrm{n}=97)$ & \\
\hline Positive hCG (\%) & & & $5 / 41(12.20)$ & $5 / 26(19.23)$ & 25/102 (24.51) & $\begin{array}{l}25 / 97 \\
(25.77)\end{array}$ & NS \\
\hline Clinical pregnancy rate (\%) & & & $4 / 41(9.76)$ & 4/26 (15.38) & $17 / 102(16.67)$ & $\begin{array}{l}17 / 97 \\
(17.53)\end{array}$ & NS \\
\hline $\begin{array}{l}\text { Ongoing pregnancy rate } \\
\text { (\%) }\end{array}$ & & & $4 / 41(9.76)$ & 4/26 (15.38) & 14/102 (13.72) & $\begin{array}{l}14 / 97 \\
(14.43)\end{array}$ & NS \\
\hline \multicolumn{8}{|l|}{ Pregnancy loss } \\
\hline Miscarriage & & & \multicolumn{2}{|c|}{0} & \multicolumn{2}{|c|}{ 3/17 (17.6) } & NS \\
\hline Ectopic pregnancy & & & \multicolumn{2}{|c|}{0} & \multicolumn{2}{|c|}{$1 / 25(4)$} & NS \\
\hline Single embryo transfer (\%) & & & \multicolumn{2}{|c|}{$13 / 26(50.0)$} & \multicolumn{2}{|c|}{ 83/97 (85.6) } & $<0.001$ \\
\hline Multiple pregnancy rate (\%) & & & \multicolumn{2}{|c|}{$1 / 4(25)$} & \multicolumn{2}{|c|}{0} & NS \\
\hline
\end{tabular}

The question whether cumulative pregnancy chances are affected by a cleavage stage embryo transfer versus blastocyst culture and blastocyst transfer based on a day 3 embryo quality evaluation remained unanswered. This study showed that such a embryology based policy mainly divides the patient population based on their ovarian response. The inherent reduced reproductive potential is illustrated by the low transferrable embryo survival rate of $46.4 \%$ compared to $65.1 \%$ as previously published in elective day 3 population in the same centre [15]. An alternative interpretation of the dataset is that a day 5 transfer is not required for a large proportion of the patients as the day 3/day 5 subgroups comparison for pregnancy rates are comparable.

In this study we used an aseptic method for the vitrification of embryos. Although the transmission of infectious diseases through cryopreservation and storage of embryos has never been reported, such possibility exists under experimental conditions $[7,16]$. Therefore, the application of hermetically sealed containers or secondary enclosure (for cryovials and open vitrification systems) is suggested [16]. The hermetically sealed device used in this study has the advantage that both vitrification and storage are perfomed under 'closed' conditions.
Aseptical vitrification using open devices would require sterilization of liquid nitrogen and a secondary enclosure for storage [17].

For the vast majority of patients, the single embryo transfer policy was based on the Belgian law imposing a SET policy in women less than 36 years [18]. No strict SET policy was applied in the thawed embryo transfer cycles, with significantly more DET (double embryo transfer) in the frozen day 3 embryo transfers. The higher proportion of DET in the frozen day 3 cycles (50\%) as compared to the frozen blastocyst cycles (14.6\%) can be explained by a lower expected implantation rate and resulted in a similar ongoing pregnancy rate per transfer (15.38\% vs. $14.43 \%)$. The p value for the subject specific clinical pregnancy rate comparing the day 3 and day 5 embryo transfer reaches 0.05 , although no difference has been observed in the pregnancy rate per transfer. The high subject specific pregnancy rate in the day 5 group appears to be due to the significantly higher transferrable survival rate and the embryo transfers performed.

Although no differences were observed in patients' and treatment characteristics, the retrospective design remains a weakness. The allocation of patients to one or the other group is based on the view of the individual 
Table 6 Summary and projected pregnancy rate compensating for the double counting of pregnancies in case a subject has more than one pregnancy

\begin{tabular}{|c|c|c|c|c|}
\hline & eSET with elective day 5 transfer & eSET with t & $\begin{array}{l}\text { ransfer day } 3 \text { or } \\
5\end{array}$ & Statistical significance \\
\hline No. of fresh cycles & 341 & & 249 & \\
\hline No. of thawed cycles & 188 & & 143 & \\
\hline Subjects with fresh or thawed clinical pregnancy & 147 & & 100 & \\
\hline Subjects with fresh or thawed ongoing pregnancy & 142 & & 91 & \\
\hline Number of embryos unthawed ${ }^{1}$ & 38 & & 98 & \\
\hline Clinical pregnancy rate thawed embryos & 26/286 (9.09) & $21 / 2$ & $29(9.17)$ & NS \\
\hline Ongoing pregnancy rate thawed embryos & 24/286 (8.39) & $18 / 2$ & $29(7.86)$ & NS \\
\hline Projected number subjects with clinical pregnancy ${ }^{2}$ & 3.45 & 9.68 & $1.24+8.44)$ & \\
\hline Projected number subjects with a ongoing pregnancy ${ }^{2}$ & 3.19 & 8.20 & $1.24+6.96)$ & \\
\hline Projected cumulative clinical pregnancy rate ${ }^{3}$ & $150.45 / 341(44.12)$ & 109.68 & $249(44.04)$ & NS \\
\hline \multirow[t]{2}{*}{ Projected cumulative ongoing pregnancy rate ${ }^{3}$} & $145.19 / 341(42.58)$ & $99.20 /$ & $249(39.84)$ & NS \\
\hline & & Day 3 & Day 5 & \\
\hline No. of fresh cycles & & 135 & 102 & \\
\hline No. of thawed cycles & & 41 & 102 & \\
\hline Subjects with fresh or thawed clinical pregnancy & & 43 & 57 & \\
\hline Subjects with fresh or thawed ongoing pregnancy & & 40 & 51 & \\
\hline Number of embryos unthawed ${ }^{2}$ & & 26 & 72 & \\
\hline Clinical pregnancy rate thawed embryos & & $4 / 84(4.76)$ & $17 / 145(11.72)$ & NS \\
\hline Ongoing pregnancy rate thawed embryos & & $4 / 84(4.76)$ & 14/145 (9.66) & NS \\
\hline Projected number subjects with clinical pregnancy & & 1.24 & 8.44 & \\
\hline Projected number subjects with a ongoing pregnancy & & 1.24 & 6.96 & \\
\hline
\end{tabular}

${ }^{1}$ Number of embryos unthawed for subjects with no fresh or thawed pregnancy.

${ }^{2}$ Projected from the unthawed material among subjects without a fresh or thawed pregnancy, assuming that the pregnancy rate will be the same as for the already thawed material.

${ }^{3}$ Fresh pregnancies, thawed pregnancies and projected number of pregnancies/number of subjects.

physician in the centre and the preferences of the patient. Establishing a prospective randomized controlled trial will generate anxiety amongst patients and clinicians. This retrospective data will be reassuring for centers wishing to investigate this subject further.

\section{Acknowledgements}

The authors wish to thank Walter Meul for his kind assistance in the data retrieval.

\section{Authors' contributions}

DS conceived of the study, performed the statistical analysis and wrote the manuscript. LVL coordinated the laboratory aspects of embryo cryopreservation and participated in the outcome evaluation. EVDA coordinated the laboratory aspects of embryo cryopreservation. MC helped to draft the manuscript. GV helped to draft the manuscript. PD conceived of the study and participated in its design and coordination and helped to draft the manuscript. All authors read and approved the final manuscript.

\section{Competing interests}

The authors declare that they have no competing interests.

Received: 21 December 2010 Accepted: 5 May 2011

Published: 5 May 2011

\section{References}

1. Bergh C: Single embryo transfer: a mini-review. Hum Reprod 2005, 20:323-327, Bielanski A, Nadin-Davis S, Sapp T, Lutze-Wallace C: Viral contamination of embryos in liquid nitrogen. Cryobiology 2000, 40:110-116..
2. Thurin A, Hausken J, Hilensjö T, Jablonowska B, Pinborg A, Strandell A, Bergh C: Elective single-embryo transfer versus double-embryo transfer in in vitro fertilization. N Engl J Med 2004, 351:2392-2402.

3. Jones $H$, Veeck $L$, Muasher $S$ : Cryopreservation: the problem of evaluation. Hum Reprod 1995, 10:2136-2138.

4. Papanikolaou E, Camus M, Kolibianakis E, Van Landuyt L, Van Steirteghem A, Devroey P: In vitro fertilization with single blastocyst-stage versus single cleavage-stage embryos. N Engl J Med 2006, 354:1139-1146.

5. Papanikolaou E, Kolibianakis $E$, Tournaye $H$, Venetis $C$, Fatemi $H$, Tarlatzis $B$, Devroey P: Live birth rates after transfer of equal number of blastocysts or cleavage-stage embryos in IVF. A systematic review and metaanalysis. Hum Reprod 2008, 23:91-99.

6. Papanikolaou E, D'haeseleer E, Verheyen G, Van de Velde H, Camus M, Van Steirteghem A, Devroey P, Tournaye H: Live birth rate is significantly higher after blastocyst transfer than after cleavage-stage embryo transfer when at least four embryos are available on day 3 of embryo culture. A randomized prospective study. Hum Reprod 2005, 20:3198-3203.

7. Bielanski A, Bergeron H, Lau P, Devenish J: Microbial contamination of embryos and semen during long term banking in liquid nitrogen. Cryobiology 2003, 46:146-152.

8. Tedder R, Zuckermann M, Goldstone A, Hawkins A, Fielding A, Briggs E, Irwin D, Blair S, Gorman A, Patterson K, Linch D, Heptonstall J, Brink N: Hepatitis B transmission from contaminated cryopreservation tank. The Lancet 1995, 346:137-140.

9. Van Landuyt L, De Vos A, Joris H, Verheyen G, Devroey P, Van Steirteghem A: Blastocyst formation in IVF versus ICSI cycles: influence of the fertilization procedure. Fertil Steril 2005, 83:1397-1403.

10. Schoolcraft WB, Gardner DK, Lane M, Schlenker T, Hamilton F, Meldrum DR: Blastocyst culture and transfer: analysis of results and parameters affecting outcome in two in vitrofertilization programs. Fertil Steril 1999, 72:604-609. 
11. Van der Auwera I, Debrock S, Spiessens C, Afschrift H, Bakelants E, Meuleman C, Meeuwis L, D'hooghe T: A prospective randomized study: day 2 versus day 5 embryo transfer. Hum Reprod 2002, 17:1507-1512.

12. Guerif $F$, Lemseffer M, Bidault $R$, Gasnier $O$, Saussereau M, Cadoret $V$, Jamet C, Royere D: Single day 2 embryo versus blastocyst-stage transfer: a prospective study integrating fresh and frozen embryo transfers. Hum Reprod 2009, 24:1051-1058.

13. Loutradi K, Kolibianakis E, Venetis C, Papanikolaou E, Pados G, Bontis I, Tarlatzis B: Cryopreservation of human embryos by vitrification or slow freezing: a systematic review and meta-analysis. Fertil Steril 2008, 90:186-193.

14. Van den Abbeel E, Camus M, Verheyen G, Van Waesberghe L, Devroey P, Van Steirteghem A: Slow controlled-rate freezing of sequentially cultured human blastocysts: an evaluation of two freezing strategies. Hum Reprod 2005, 20:2939-2945.

15. Van den Abbeel, Van Steirteghem A: Zona pellucida damage to human embryos after cryopreservation and the consequences for their blastomere survival and in-vitro viability. Hum Reprod 2000, 15:373-378.

16. Bielanski A, Vajta G: Risk of contamination of germplasm during cryopreservation and cryobanking in IVF units. Hum Reprod 2009, 24:2457-2467.

17. Parmegiani L, Accorsi A, Cognigni G, Bernardi S, troilo E, Fillicori M: Sterilization of liquid nitrogen with ultraviolet irradiation for safe vitrification of human oocytes and embryos. Fertil Steril 2010.

18. Van Landuyt L, Verheyen G, Tournaye H, Camus M, Devroey P, Van Steirteghem A: New Belgian embryo transfer policy leads to sharp decrease in multiple pregnancy rate. Reprod Biomed online 2006, 13:765-771.

doi:10.1186/1477-7827-9-60

Cite this article as: Stoop et al: Should a single blastocyst transfer policy be a clinical decision or should it depend on the embryological evaluation on day 3? Reproductive Biology and Endocrinology 2011 9:60.

\section{Submit your next manuscript to BioMed Central and take full advantage of:}

- Convenient online submission

- Thorough peer review

- No space constraints or color figure charges

- Immediate publication on acceptance

- Inclusion in PubMed, CAS, Scopus and Google Scholar

- Research which is freely available for redistribution

Submit your manuscript at www.biomedcentral.com/submit
Biomed Central 